\title{
Coexistence of Wireless Technologies in Medical Scenarios
}

\author{
Heikki Karvonen, Matti Hämäläinen and Jari Iinatti \\ Centre for Wireless Communications \\ University of Oulu \\ Oulu, Finland \\ \{heikki.karvonen, matti.hamalainen, jari.iinatti\}@oulu.fi
}

\author{
Carlos Pomalaza-Ráez \\ Department of Electrical and Computer Engineering \\ Purdue University \\ Fort Wayne, Indiana, USA \\ cpomalaz@purdue.edu
}

\begin{abstract}
The goal of this paper is to provide a comprehensive overview of the coexistence nature of wireless technologies most likely to be found in medical scenarios' environment. The diversity and number of these technologies is increasing constantly leading to potential interference problems and performance degradation of wireless medical applications which are expected to become popular in 5G systems. The industrial, scientific and medical (ISM) bands in the $2.4 \mathrm{GHz}$ are already very crowded to the point that the location and use of medical devices have to take into account the pervasive presence of other wireless devices operating in that region of the spectrum. A temporary solution is to use more the $5 \mathrm{GHz}$ bands currently not so heavily utilized. This scenario will change in the near future as unlicensed long-term evolution (LTE) solutions such as MulteFire start operating in those bands. This paper provides a summary of the wireless technologies and devices present in hospitals and other medical care scenarios. It also provides recommendations for the rational share of the spectrum in those scenarios.
\end{abstract}

Keywords-spectrum management; unlicensed band; licensed band; wireless body area network; healthcare applications; $5 G$ system;

\section{INTRODUCTION}

The use of wireless communications systems has increased considerably in hospitals and health care scenarios. A recent report [1] estimates that the market for wireless medical devices is expected to show about $25.1 \%$ market growth over the next five years. The worldwide market has the potential to expand from $\$ 3.6$ billion in 2015 to about $\$ 11.0$ billion by 2020. These days it is common to find in hospitals a variety of wireless devices that monitor patients' vital signs, that help locate doctors, nurses, and equipment, and that are used to communicate among care givers. Patients, their visitors, and the hospital personnel continuously use cellular communications and wireless local area networks (WLAN). The presence of microwaves and wireless devices that monitor the building environment, e.g. thermostats, lighting, also adds to the electromagnetic radiation present in medical scenarios.

Electromagnetic compatibility or more specific coexistence of wireless devices and other sources of electromagnetic radiation must be carefully monitored and controlled in hospitals and other health care environments to ensure the safety of the patients and their care givers. The scope of this paper is to survey relevant features of the wireless technologies present in this type of scenarios, in particular their spectrum occupancy, power levels and data rates.

Section II provides a summary of wireless technologies that have been specifically designed for medical applications or that are most likely to be used by medical devices. Section III summarizes other wireless technologies that are present in medical scenarios that in some cases complement those described in Section II but in other cases compete with them for spectrum occupancy. Section IV discusses relevant technical specifications of medical devices currently in the market. Coexistence among wireless technologies most likely to be found in medical scenarios and recommendations are discussed in Section V.

\section{ON- AND IN-BODY DEVICES/SYSTEMS}

The large majority of wireless devices used in medical applications operate in the unlicensed bands of the spectrum, in particular those bands that have been designated for industrial, scientific, and medical (ISM) communication purposes. They are unlicensed but they are still regulated, e.g. in Europe by CEPT (European Conference of Postal and Telecommunications Administrations) [2]. Several well established technologies, such as Bluetooth [3], ZigBee [4], radio-frequency identification (RFID) [5] and IEEE Std. 802.11 [6] operate in the ISM bands. Some of these bands are globally designated as being unlicensed, e.g. $2.400 \mathrm{GHz}$ to $2.483 \mathrm{GHz}$ with some variations from region to region. But all bands are not common, for example in Europe the ISM 863$870 \mathrm{MHz}$ band is designated for SRD but it is not in the US (the closest is the 902-928 MHz band).

In the next paragraphs a brief summary of wireless technologies that are commonly found in medical scenarios is presented. Most of them are on-body devices but recently there has been an increase of in-body devices, as well as devices used by medical device providers. The main characteristics of the technologies described in this section is shown in Table 1.

Bluetooth is a wireless technology that has been around for over twenty years and has gone through several iterations, each improving or expanding the features of the previous one. 
Table 1. Characteristics of wireless body area network (WBAN) technologies.

\begin{tabular}{|c|c|c|c|c|c|}
\hline & Frequency Band & Channel bandwidth & Modulation & Bit Rate & Transmit Power \\
\hline BLE [3] & $2401-2481 \mathrm{MHz}$ & $2 \mathrm{MHz}$ & GFSK & $1 \mathrm{Mbps}$ & -20 to $10 \mathrm{dBm}$ \\
\hline Classic Bluetooth [3] & $2401.5-2480.5 \mathrm{MHz}$ & $1 \mathrm{MHz}$ & $\begin{array}{c}\text { GFSK } / \pi / 4-\mathrm{DQPSK} / \\
8 \mathrm{DPSK}\end{array}$ & $\begin{array}{l}721 \mathrm{kbps} \\
3 / 24 \mathrm{Mbps}\end{array}$ & $0 / 4 / 20 \mathrm{dBm}$ \\
\hline IEEE Std. 802.15.6 [8] & $\begin{array}{c}\text { NB: } 402-2483.5 \mathrm{MHz} \\
\text { UWB: } 3-10 \mathrm{GHz} \\
\text { HBC: } 18-26 \mathrm{MHz}\end{array}$ & $\begin{array}{c}\text { NB: } 300-1000 \mathrm{kHz} \\
\text { UWB: } 499.2 \mathrm{MHz} \\
\text { HBC: } 5.25 \mathrm{MHz}\end{array}$ & $\begin{array}{c}\text { NB: DBPSK/GMSK } \\
\text { UWB: OOK, } \\
\text { DBPSK/DQPSK, } \\
\text { BFSK } \\
\end{array}$ & $\begin{array}{l}\text { NB: } 75.9- \\
\text { 971.4 kbps } \\
\text { UWB: } 0.487- \\
15.6 \mathrm{Mbps} \\
\end{array}$ & $\begin{array}{c}-16 \mathrm{dBm} /-40 \mathrm{dBm} \text { at } \\
403.65 \mathrm{MHz} \\
-10 \mathrm{dBm} \text { at other } \\
\text { frequencies } \\
\end{array}$ \\
\hline ETSI SmartBAN [9] & $2401-2481 \mathrm{MHz}$ & $2 \mathrm{MHz}$ & GFSK & $0.2-1 \mathrm{Mbps}$ & max. $10 \mathrm{dBm}$ EIRP \\
\hline $\mathrm{ANT} / \mathrm{ANT}+[10]$ & $\begin{array}{l}2400-2524 \mathrm{MHz} \\
\text { ANT+: } 2547 \mathrm{MHz}\end{array}$ & $1 \mathrm{MHz}$ & GFSK & $1 \mathrm{Mbps}$ & Up to $4 \mathrm{dBm}$ \\
\hline Sensium [11] & $868 / 915 \mathrm{MHz}$ & $200 \mathrm{kHz}$ & BPSK & $50 \mathrm{kbps}$ & $-7 /-10 \mathrm{dBm}$ \\
\hline Zarlink [12] & $\begin{array}{c}402 \mathrm{MHz}-405 \mathrm{MHz} \\
433 \mathrm{MHz}-434 \mathrm{MHz} \\
2.45 \mathrm{GHz} \text { for wake-up }\end{array}$ & $100-200 \mathrm{kHz}$ & $\begin{array}{l}\text { 2FSK } \\
\text { 4FSK }\end{array}$ & $\begin{array}{l}18.18 / 40 / 200 \\
/ 400 / 800 \mathrm{kbps}\end{array}$ & -3 to $-30 \mathrm{dBm}$ \\
\hline BodyLAN [13] & $2.4 \mathrm{GHz}$ & - & GFSK & $\begin{array}{c}250 \mathrm{kbps} \\
1 \mathrm{Mbps}\end{array}$ & $0 \mathrm{dbm}$ \\
\hline
\end{tabular}

A low-power version (Bluetooth 4.0), called Bluetooth Low Energy (BLE) or Bluetooth Smart was introduced in 2010. It consumes little power and can be operated with small power sources or by harvesting energy. It is becoming widely used on a large variety of medical devices, e.g. heart rate and blood pressure monitors. It operates in the $2.4 \mathrm{GHz}$ ISM band. Its medium access control (MAC) protocol uses 3 advertising channels strategically positioned to minimize interference and channel occupancy. Recent update, Bluetooth 5.0 improves further this technology's suitability for the Internet of Things (IoT) applications by increasing range, speed and broadcasting capacity.

IEEE 802.15.6 [8] and ETSI SmartBAN [9] standards are the first ones tailored specifically for body area networks that can be used in health, medical, and sport applications. The IEEE Std. 802.15.6 is intended for low-power devices to be used in wireless body area networks. This standard offers three physical (PHY) layer options: narrowband (NB), ultrawideband (UWB) and human body communications (HBC). The MAC layer for this standard has three different modes and thus it offers flexibility for operation in different scenarios. The ETSI SmartBAN standard is more recent than IEEE Std. 802.15.6. The frequency of operation of this standard is between $2.401 \mathrm{GHz}$ and $2.481 \mathrm{GHz}$ with $2 \mathrm{MHz}$ spacing, i.e. similar to the classic Bluetooth. This protocol uses separate channels for data and control messages and like the IEEE Std. 802.15.6 it has different channel access modes [9].

ANT/ANT $+[10]$ is low-power proprietary protocol designed for low data rate sensor and body area networks that can be configured in different topologies, e.g. peer-to-peer, star, and mesh. It operates in the $2.400-2.524 \mathrm{GHz}$ band, the ANT + devices operates in the $2.457 \mathrm{GHz}$ frequency. The ANT channels are $1 \mathrm{MHz}$ wide. The protocol is time division multiple access (TDMA) based with channel hopping capabilities. The flexibility features of this protocol allow ANT devices to adapt to hostile conditions by changing the amount of control overhead depending on the amount of experienced interference.

Sensium [11] is a proprietary protocol that operates in the $868 \mathrm{MHz}$ and $915 \mathrm{MHz}$ frequency regions. The SensiumVitals system is designed for the monitoring of patient's vital signs relying on a radio chip in compliance with the IEEE Std. 802.15.6 narrowband PHY layer.

Zarlink [12] is a low-power proprietary transceiver designed for medical implantable applications. It operates in $402-405 \mathrm{MHz}$ and 433 - $434 \mathrm{MHz}$ bands. It can operate in both implants and in an on-body hub devices. When operating in an implant device, the radio is in a sleep mode most of the time and it makes use of a wake-up radio that operates in the $2.4 \mathrm{GHz}$ band or it wakes up directly using a clock mechanism within the implantable device.

BodyLAN [13] is a proprietary technology that operates in the $2.4 \mathrm{GHz}$ band. It is focused on fitness equipment and devices but is also deployed in medical devices and consumer electronics. It relies on transmissions of short burst durations, very low duty-cycle, and Gaussian frequency-shift keying (GFSK) modulation. These features minimize the interference from IEEE Std. 802.11g orthogonal frequency-division multiplexing (OFDM) / direct sequence spread spectrum (DSSS) packets.

\section{COMPLEMENTARY/COMPETING WIRELESS TECHNOLOGIES}

This section has a description of wireless technologies that are likely to be found in medical scenarios. These technologies can be complementing or enhancing the functions of the technologies described in Section II, but in other cases they are performing non-medical related functions, e.g. building environmental control, occupying the same spectrum bands used by medical devices and potentially interfering with their performance. A brief description of several of these technologies is given next.

The IEEE 802.11 [6] is an ever evolving set of PHY and MAC layer standards specifications for the implementation of WLAN communications. The original version of this standard was released in 1997. Ever since then it has been revised increasing the range of its features, in particular the bit rates. When operating in the $2.4 \mathrm{GHz}$ frequency bands $(802.11 \mathrm{~b} / \mathrm{g})$ it runs into a lot of completion with other wireless devices and even electric/electronic appliances, e.g. microwave ovens. 
Table 2. Characteristics of technologies in hospital environment.

\begin{tabular}{|c|c|c|c|c|c|}
\hline & Frequency Band & Channel BW & Modulation & Bit Rate & Tx Power \\
\hline IEEE Std. 802.15 .4 & $\begin{array}{c}868 / 915 \mathrm{MHz} \\
2.4 \mathrm{GHz} \\
\text { UWB: } \\
249.6 \text { to } 749 \mathrm{MHz} \\
3.1 \text { to } 4.8 \mathrm{GHz} \\
5.8 \text { to } 10.6 \mathrm{GHz} \\
\end{array}$ & $\begin{array}{c}1(868 \mathrm{MHz}) \\
10(915 \mathrm{MHz}) \\
16(2.4 \mathrm{GHz}) \\
\text { UWB: } 500 \text { to } 1354 \mathrm{MHz}\end{array}$ & $\begin{array}{c}\text { BPSK } \\
\text { O-QPSK } \\
\text { UWB: BPM-BPSK }\end{array}$ & $\begin{array}{c}20 \mathrm{kbps} \\
40 \mathrm{kbps} \\
250 \mathrm{kbps} \\
\text { UWB: } 0.11-27 \mathrm{Mbps}\end{array}$ & -32 to $0 \mathrm{dBm}$ \\
\hline IEEE Std. 802.11a & $5 \mathrm{GHz}$ & $20 \mathrm{MHz}$ & OFDM & $6-54 \mathrm{Mbps}$ & $14-20 \mathrm{dBm}$ \\
\hline IEEE Std. $802.11 \mathrm{~b} / \mathrm{g}$ & $2.4 \mathrm{GHz}$ & $22 \mathrm{MHz}$ & DSSS/OFDM & $1-54 \mathrm{Mbps}$ & $20 \mathrm{dBm}$ \\
\hline IEEE Std. $802.11 \mathrm{n}$ & $\begin{array}{c}2.4 \mathrm{GHz} \\
5 \mathrm{GHz}\end{array}$ & $\begin{array}{l}20 \mathrm{MHz} \\
40 \mathrm{MHz}\end{array}$ & MIMO-OFDM & $\begin{array}{c}7-288 \mathrm{Mbps} \\
15-600 \mathrm{Mbps}\end{array}$ & $\begin{array}{l}16-20 \mathrm{dBm} \\
14-20 \mathrm{dBm}\end{array}$ \\
\hline IEEE Std. 802.11ac & $5 \mathrm{GHz}$ & $\begin{array}{c}40 \mathrm{MHz} \\
80 \mathrm{MHz} \\
160 \mathrm{MHz} \\
\end{array}$ & $\begin{array}{l}\text { OFDM } \\
\text { M-QAM }\end{array}$ & $\begin{array}{c}500 \mathrm{Mbps}-1 \mathrm{Gbps} \\
\max 6.93 \mathrm{Gbps}\end{array}$ & $20 \mathrm{dBm}$ \\
\hline RFID & $\begin{array}{c}135 / 13.56 \mathrm{kHz} \\
2.45 / 5.8 \mathrm{GHz}\end{array}$ & $\begin{array}{c}0.5 \mathrm{MHz} \text { when in } 2.45 \\
\mathrm{GHz}\end{array}$ & $\begin{array}{l}\text { ASK - OOK when } \\
\text { in } 2.45 \mathrm{GHz} \\
\end{array}$ & $\begin{array}{c}30 \text { to } 40 \mathrm{kbps} \text { when in } \\
2.45 \mathrm{GHz} \\
\end{array}$ & $\begin{array}{l}\sim 0 \mathrm{dBm} \text { for active } \\
\text { RFIDs at } 2.45 \mathrm{GHz}\end{array}$ \\
\hline LoRa & $150 \mathrm{MHz}-1 \mathrm{GHz}$ & 125,250 and $500 \mathrm{kHz}$ & $\begin{array}{l}\text { Chirp spread } \\
\text { spectrum }\end{array}$ & $0.3-50 \mathrm{kbps}$ & $\max .14 \mathrm{dBm}$ \\
\hline MulteFire & $\begin{array}{c}5 \mathrm{GHz} \text { ISM band } \\
3.5 \mathrm{GHz} \text { (US) }\end{array}$ & $20 \mathrm{MHz}$ & OFDM, M-QAM & Up to $400 \mathrm{Mbps}$ & $20 \mathrm{dBm}$ \\
\hline LTE-U & LTE and unlicensed bands & $20 \mathrm{MHz}$ & OFDM, M-QAM & $300 \mathrm{Mbps}$ & $20 \mathrm{dBm}$ \\
\hline NB-IoT (LTE) & LTE bands & $180 \mathrm{kHz}$ & OFDM/SC-FDMA & $20-250 \mathrm{kbps}$ & $23 \mathrm{dBm}$ \\
\hline LTE-M & LTE bands & $1.08 \mathrm{MHz}$ & OFDM/SC-FDMA & Up to $1 \mathrm{Mbps}$ & $23 \mathrm{dBm}$ \\
\hline
\end{tabular}

The $802.11 \mathrm{a}$ version of the standard operates in the $5 \mathrm{GHz}$ region avoiding the congested $2.4 \mathrm{GHz}$ region. It uses 52subcarriers (OFDM) with a maximum of 54 Mbps. 802.11g operates in the $2.4 \mathrm{GHz}$ region but it uses the same type of OFDM scheme as $802.11 \mathrm{a}$ and is able to provide a maximum rate of 54 Mbps. Substantial increase in bit rates, reliability, and throughput, are achieved with the $802.11 \mathrm{n}$ version. The use of multiple antennas, an efficient MAC layer, and a wider channel $(40 \mathrm{MHz})$ allows for a theoretical maximum of 600 Mbps. 802.11n can operate in the $2.4 \mathrm{GHz}$ and 5 (5.725-5.850) $\mathrm{GHz}$ frequency ranges. A recent (2013) version, 802.11ac, can provide a maximum data rate of $6.75 \mathrm{Gbps}$. It has the option to operate on $40 \mathrm{MHz}, 80 \mathrm{MHz}$, and $160 \mathrm{MHz}$ bandwidths in the $5 \mathrm{GHz}$ ISM band.

For building automation and environment conditions monitoring, the ZigBee [4] and LoRa [14] solutions might become common in future deployments. ZigBee is based on IEEE Std. 802.15.4 and most of the solutions are operating at congested $2.4 \mathrm{GHz}$ band. LoRa technology is operating at sub$\mathrm{GHz}$ bands. 3GPP group is also actively developing IoT solutions, such as NB-IoT and LTE-M which will operate in licensed cellular bands [15].

RFID devices are being used widely in all type of environments, including hospitals and medical care facilities. They use several regions of the spectrum, e.g. $135 \mathrm{kHz}, 13.56$ $\mathrm{MHz}, 2.45 \mathrm{GHz}$ and $5.8 \mathrm{GHz}$. Of particular interest are the tags that work in the $2.45 \mathrm{GHz}$ and $5.8 \mathrm{GHz}$ where many of the devices used in medical scenarios are also used. Interference of RFIDs with medical equipment has been found to be a direct function of the distance between the tag and the medical devices.

There are several competing proposals to bring LTE performance to the unlicensed $5 \mathrm{GHz}$ region of the spectrum in order to increase the capacity of cellular networks. These proposals have originated, to this date, contradictory reports about the coexistence of LTE-U (unlicensed) and Wi-Fi networks [16], [17]. An approach to improve the capacity is to combine the use of unlicensed spectrum with licensed bands (LTE License Assisted Access (LAA) and LTE - Wi-Fi link aggregation (LWA)). There has been a flurry of proposals in the literature aiming to improve the coexistence of LTE and Wi-Fi networks. MulteFire is a new technology which enables to use LTE technology solely in unlicensed band, particularly at $5 \mathrm{GHz}$ [18]. A summary of the main features of the technologies described in this section are shown in Table 2.

\section{COMMERCIALly APPROVED MEDICAL DEVICES}

It is useful to have an inventory of what is the current state of the art regarding commercial devices currently in use at medical care locations. The majority of these devices are onbody type, i.e. they are deployed on the body or within very close proximity of the human body. In-body devices are becoming more common but communication environment is more challenging and it takes a longer time to have them tested and approved, thus they are not numerous in the market. Finally, there are on-body medical wireless devices that are used by medical providers to assist them when carrying out their work, e.g., drawing blood. Table 3 shows a representative sample of commercially available wireless medical devices. From Table 3 it is apparent that, other than implant medical devices, the majority of the current commercially available wireless medical devices use Bluetooth Low Energy technology. BLE operates in the crowded $2.4 \mathrm{GHz}$ frequency bands. It is expected than many future medical devices will operate in $5 \mathrm{GHz}$ frequency bands to improve their wireless connectivity. It should be noted that because of health considerations not all medical devices can operate at those frequencies not even at the $2.4 \mathrm{GHz}$, e.g., pacemakers. 
Table 3. Examples of commercial medical devices.

\begin{tabular}{|c|c|c|c|}
\hline Type & Vendor/Device & Measures & Wireless technology \\
\hline \multirow{15}{*}{ On-Body } & iHealth/BP7 ${ }^{1}$ & Blood Pressure & Bluetooth V3.0+EDR Class 2 SPP \\
\hline & Trisa/Cardio Pro $4.0^{2}$ & Blood Pressure & Bluetooth \\
\hline & Withings $^{3}$ & Blood Pressure & Bluetooth, BLE \\
\hline & Nonin $/ 3230^{4}$ & Pulse Oximeter & BLE \\
\hline & iHealth/PO3 ${ }^{5}$ & Pulse Oximeter & BLE \\
\hline & Visiomed/BW-OX1 $^{6}$ & Pulse Oximeter & BLE \\
\hline & LIFESYNC $^{7}$ & Electrocardiogram & Bluetooth \\
\hline & QardioCore $^{8}$ & Electrocardiogram & BLE \\
\hline & Emotiv/EPOC+ ${ }^{9}$ & Electroencephalogram & BLE, proprietary in the $2.4 \mathrm{GHz}$ \\
\hline & Medtronics/MiniMed 640G ${ }^{\mathbf{1 0}}$ & Blood sugar level/Insulin pump & 802.15 .4 (proprietary data format) \\
\hline & Quell ${ }^{11}$ & Pain management & BLE \\
\hline & Eko Core ${ }^{12}$ & Heart, lung sounds (stethoscope) & BLE \\
\hline & VitalConnect $^{13}$ & $\begin{array}{c}\text { ECG, heart \& respiratory rate, temperature, } \\
\text { fall detection }\end{array}$ & BLE \\
\hline & Moticon/OpenGo ${ }^{14}$ & $\begin{array}{c}\text { Food dynamics (pressure distribution, } \\
\text { weight, balance, and motion }\end{array}$ & BLE \\
\hline & BioRadio $^{15}$ & $\begin{array}{c}\text { ECG, EEG, EMG, respiration, SpO2, } \\
\text { Temperature, Spirometry, \& Galvanic Skin } \\
\text { Response (GSR) }\end{array}$ & BLE \\
\hline Near-Body & Dräger Infinity (M300) ${ }^{16}$ & ECG, heart rate, pulse oximetry & Wi-Fi \\
\hline \multirow{2}{*}{ In-Body } & Senseonics/Eversense ${ }^{17}$ & Continuous glucose monitoring & 13.56 MHz inductive link, on-body BLE \\
\hline & Boston Scientific/Vitalio ${ }^{18}$ & Pacemaker & $916.5 \mathrm{MHz}$ \\
\hline $\begin{array}{c}\text { On-body medical } \\
\text { provider }\end{array}$ & Evena/Eye-On Glassess $3.0^{19}$ & Vascular multispectral imaging & Bluetooth, Wi-Fi, 3G Wireless \\
\hline
\end{tabular}

\section{COEXISTENCE AND ReCOMMENDATIONS}

Fig. 1 summarizes the locations, in the frequency spectrum, of the common wireless technologies. It is apparent that a large variety of technologies are using unlicensed ISM bands, the $2.4 \mathrm{GHz}$ band being the most crowded.

The majority of the commercially available state-of-the-art on-body devices use BLE. This technology operates in the 2.4 $\mathrm{GHz}$ and has features that make it, to a certain degree, resilient to interference. There are a few medical and wellness care devices using the IEEE Std. 804.15.4 [7]. This standard has been shown consistently, via experiments and analytical studies, to be vulnerable to $\mathrm{Wi}-\mathrm{Fi}$ in the $2.4 \mathrm{GHz}$ region. Special attention must be given to the physical location of the sensor nodes, the frequency channels they are using, and the location of the Wi-Fi routers.

In scenarios where devices using Bluetooth and 802.15.4 are operating in the same physical space experimental studies have shown that Bluetooth is more vulnerable to 802.15.4 than vice versa [19]. The most likely explanation is the higher channel occupancy of 802.15.4. Properly control of duty cycles and power levels of 802.15.4 devices, and Bluetooth / BLE frequency hopping, can minimize this interference.

Recent experimental measurements at hospitals [20] show that for the time being there is still sufficient available spectrum in the $2.4 \mathrm{GHz}$ region for the various medical wireless devices and other wireless systems to work at their normal levels without any intervention or control, even at peak hours. However, the fact that the unlicensed bands in the 2.4 $\mathrm{GHz}$ are becoming more and more congested has led to efforts to share those bands in a more rational way instead of reactive ad-hoc solutions. For medical scenarios, proposals such as the one described in [21] are worth to consider. They propose a generic protocol stack that allows for different wireless technologies with different higher level stacks. In simple terms, it recognizes the presence of heterogeneous networks. It attempts to use common features and then implements a cognitive procedure for the layers to share information available to each particular protocol stack. In addition, it must be noted that recently defined spectrum slots, around the 2.4 $\mathrm{GHz}$ ISM band, have been designated for medical communication in Europe $(2.4835-2.5 \mathrm{GHz})$ and in the US (2.36-2.4 GHz). These spectrum slots may play an important role in wireless medical applications in the $2.4 \mathrm{GHz}$ region.

The ISM band at $5 \mathrm{GHz}$ is currently much less crowded than the $2.4 \mathrm{GHz}$ band. However, state-of-the-art Wi-Fi base stations are now able to operate both in $2.4 \mathrm{GHz}$ and $5 \mathrm{GHz}$ ISM bands and therefore more traffic is expected to appear in the $5 \mathrm{GHz}$ bands. In addition, new wireless communications technologies such as MulteFire have been recently launched to operate in the $5 \mathrm{GHz}$ band. The use of the $5 \mathrm{GHz}$ frequency bands to extend LTE services has created new coverage opportunities but also concerns about the coexistence of LTE with Wi-Fi services [17]. There are reports highlighting the negative impact of LTE on Wi-Fi, but there are also reports concluding that such impact is very small. A more realistic solution moving forward is that both sides of this issue, the Wi-Fi industry and the telco operators, will have to adapt their standards to share the spectrum in a constructive way. The use of LTE in the $5 \mathrm{GHz}$ will be go a long way in solving the problem of continuously monitoring patients as they move from one place to another since devices will be able to
${ }^{1} \mathrm{http}: / /$ bit.ly/2j4utuZ
${ }^{2} \mathrm{http}: / /$ bit.ly/21KH37s
${ }^{3} \mathrm{http} / /$ bit.ly/2ln4gvu
${ }^{4} \mathrm{http}: / /$ bit.ly/2ml1SCY
${ }^{5} \mathrm{http} / /$ bit.ly/2lu7OKD

${ }^{6}$ http://bit.ly/2m6Qa2L

${ }^{7} \mathrm{http}: / /$ bit.ly/217598Y

${ }^{8} \mathrm{http} / / /$ bit.ly/1 VpTe5k

${ }^{9} \mathrm{http}: / /$ bit.ly/2ln8rYg

${ }^{10} \mathrm{http}: / /$ bit.ly/1StHlcr
${ }^{11} \mathrm{http}: / /$ bit.ly/2luk1iq

$12 \mathrm{http}: / /$ bit.ly/22mp2rY

$13 \mathrm{http} / / /$ bit.ly/2lnaaN4

${ }^{14} \mathrm{http}: / /$ bit.ly/2mbpjzt

$15 \mathrm{http}: / /$ bit.ly/2kYnu7g
${ }^{16} \mathrm{http}: / /$ bit.ly/2kL9ank
${ }^{17} \mathrm{http}: /$ bit.ly/2kL01va
${ }^{18} \mathrm{http}: / /$ bit.ly/2luf6hG
${ }^{19} \mathrm{http}: / /$ bit.ly/2lFbxqT 


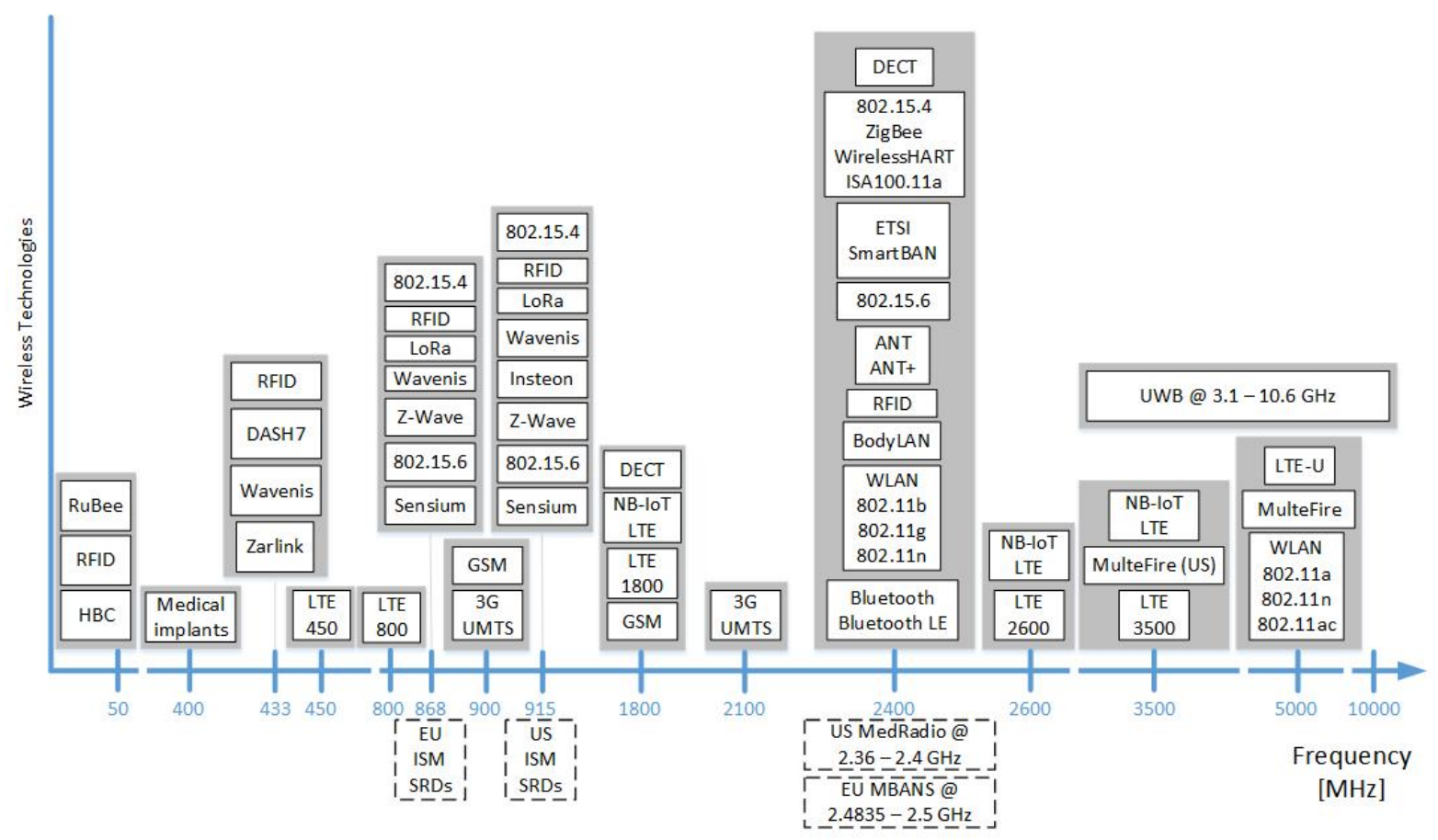

Fig. 1. Common wireless techniques at different frequencies.

leverage well tested LTE mobility features.

Spectrum sensing techniques developed for cognitive radio could be used to dynamically sense the spectrum in a medical scenario and a selection of free channel could be made. However, this solution is not comprehensive and suitable for time critical applications since the channel access cannot be guaranteed. Here it is recommended that for a privately owned environment, like hospital area, the frequency spectrum should be managed in a centralized manner. Not only information from dynamic spectrum sensing devices, but also from geolocation databases should be used to aid in the proper sharing of the spectrum. The information collected can be processed to optimize a priority-based spectrum sharing process in order to guarantee minimum communication requirements of critical applications in a medical care facility. The spectrum coordinator could for example, restrict the bandwidth available to visitors in certain areas in order to guarantee that the medical devices can get enough bandwidth to meet their functions.

\section{ACKNOWLEDGMENT}

This work has been partially funded by the Finnish Funding Agency for Innovation (Tekes) through the WILLE project.

\section{REFERENCES}

[1] Innovative Research and Products (iRAP), "Wireless medical devices: a global technology, industry and market analysis", Market Research report, Feb. 2016.

[2] CEPT ECC, "ERC Recommendation 70-03: Relating to the use of Short Range Devices (SRD)" February 2017.

[3] Bluetooth SIG, https://www.bluetooth.com/

[4] ZigBee Alliance, http://www.zigbee.org/
[5] RAIN RFID Allicance, http://rainrfid.org/

[6] IEEE 802.11 LAN Working Group, http://www.ieee802.org/11/

[7] IEEE Standard for Low-Rate Wireless Networks,"IEEE Std 802.15.42015 (Revision of IEEE Std 802.15.4-2011)”' April 22, 2016.

[8] IEEE Std. 802.15.6: IEEE Standard for Local and metropolitan area networks-Part 15.6:Wireless Body Area Networks. Standard, The Institute of Electrical and Electronics Engineers, Inc., 2012.

[9] M. Hämäläinen et al., "ETSI TC SmartBAN: Overview of the wireless body area network standard," International Symposium on Medical Information and Communication Technology (ISMICT), 2015.

[10] ANT/ANT+, https://www.thisisant.com/

[11] Sensium, http://www.sensium-healthcare.com/

[12] Microsemi (2015). Available online: http://www.microsemi.com/

[13] FitSense BodyLAN wireless protocol, http://www.sonicboomwellness.com/static/wims/512e86ef761fd08b3d0 00000/bodylan-wireless-protocol.pdf

[14] LoRa Alliance, https://www.lora-alliance.org/

[15] Nokia white paper, "LTE evolution for IoT connectivity," 2017.

[16] X. Wang, T. Q. S. Quek, M. Sheng and J. Li, "Throughput and Fairness Analysis of Wi-Fi and LTE-U in Unlicensed Band," in IEEE Journal on Selected Areas in Communications, vol. 35, no. 1, pp. 63-78, Jan. 2017.

[17] J. Li, X. Wang, D. Feng, M. Sheng and T. Q. S. Quek, "Share in the Commons: Coexistence between LTE Unlicensed and Wi-Fi," in IEEE Wireless Communications, vol. 23, no. 6, pp. 16-23, December 2016.

[18] MulteFire Allicance, http://www.multefire.org/wpcontent/uploads/2016/10/72-multefire-lights-up-the-path-for-universalwireless-service.pdf

[19] R. Natarajan, P. Zand and M. Nabi, "Analysis of coexistence between IEEE 802.15.4, BLE and IEEE 802.11 in the $2.4 \mathrm{GHz}$ ISM band," IEEE IECON, October, 2016.

[20] M. Virk, R. Vuohtoniemi, M. Hämäläinen, J.-P. Mäkelä, and J. Iinatti, "Spectrum occupancy evaluations at $2.35-2.50 \mathrm{GHz}$ ISM band in a hospital environment," International Conference on Body Area Networks (BodyNets), Oct., 2014

[21] H. Fotouhi, A. Čaušević, M. Vahabi, M. Björkman, "Interoperability in heterogeneous low-power wireless networks for health monitoring systems," IEEE ICC Workshop on Convergent Internet of Things, 2016. 